\title{
A neural network approach for evaluating distribution system reliability
}

\author{
Chern-Lin Chen \\ Department of Electrical Engineering, National Taiwan University, Taipei (Taiwan) \\ Jiann-Liang Chen \\ Advanced Technology Center, Computer and Communication Research Laboratories, Industrial Technology Research Institute, \\ Chutung, Hsinchu (Taiwan)
}

(Received October 22, 1992)

\begin{abstract}
An effective method for evaluating the distribution system reliability using an artificial neural network (ANN) is proposed in this paper. The ANN is constructed according to the back-propagation learning rule which is an iterative gradient algorithm designed to adjust the interconnection weights among processing elements. Therefore, the developed ANN is used to predict the distribution system reliability using historical data. At the same time, the system average interruption frequency index (SAIFI) and the system average interruption duration index (SAIDI) of a real distribution system are computed and compared with results generated by the network method. It was found that the deviation of the results computed by the proposed approach is below $1 \%$ and the required running time on a SUN network environment is less than $2 \mathrm{~s}$. In addition, for handling the distribution system configuration changes induced by overloading or faults, the ANN approach demonstrates an advantage over the network method.
\end{abstract}

Keywords: distribution system reliability, artificial neural network, network method.

\section{Introduction}

Distribution networks operating at several voltage levels and including the networks of local or municipal utilities are the primary parts of the power systems that deliver energy from the area supply stations to the customers. From the customers' point of view, the reliability of these systems is at least as important as that of generation and transmission. Therefore, distribution system reliability evaluation is a major preoccupation of the Taiwan Power Company, in particular, in the face of emergencies and necessary reconfiguration.

Several methods [1-7] have been presented in the literature for evaluating distribution system reliability; these may be categorized into two broad groups. One is based on the solution of logic networks and the other on the solution of state-space models. Between them, these methods can accommodate most practical systems. In addition, the network methods for determining system reliability are comparatively simple and direct. But, the obvious prerequisites for using the network methods is that a logic diagram must exist for the system under investigation. In the state-space method, a system is described by its states and the possible transitions between them. Its main techniques are the solution of Markov models and the analysis of failure effects. But, the system size that can be accommodated by an algorithm based on the statespace method may be limited.

In this paper, it is proposed to solve the problem of distribution system reliability evaluation by designing an artificial neural network (ANN) which can learn from patterns encountered previously. Many types of network exist [8-10], but the research described here focuses on a threelayer feedforward network with the back-propagation learning rule $[11,12]$. The developed ANN was utilized to evaluate the distribution system reliability. Three different cases are studied on a real distribution system in North Taiwan. Results from the developed ANN are satisfactory compared with those computed by the network 
method. With sufficient learning, the ANN generates results very fast and incurs only tiny deviations. The ANN also has an advantage over the network method in computing system reliability when one or more circuit breakers open due to a fault or overload switching. Under such situations, the network method repeats the whole computation procedure because the system logic diagram differs. Yet in the ANN approach the related links are simply modified by resetting their weights.

The remaining parts of this paper are organized as follows. Section 2 gives the reasons why we use an ANN to compute distribution system reliability. The subsequent section depicts the construction of such an ANN. Test studies on a real distribution system are described in $\S 4$, and $\S 5$ concludes the study.

\section{Validation of an ANN application}

In this section, we would like to validate an ANN application for evaluating distribution system reliability by comparing its characteristics with those of other applications. Successful ANN applications have many common characteristics including the following [13]:

(1) the application domain is data-intensive and dependent upon multiple interacting parameters;

(2) the problem area is rich in historical data or examples;

(3) the data set is incomplete and utilized for specific examples;

(4) the function to determine solutions is unknown or difficult to discover.

Several classes of application amenable to an ANN approach often involve either pattern recognition or statistical mapping. The distribution system reliability evaluation is a data-intensive problem. The failure rate and restoration time of each component, the switching times of switches and breakers, the system configuration, and the load data are required as input. In addition, rich historical data also exist. Moreover, conventional solution methods are time-consuming.

\section{Construction of an ANN network}

\subsection{Three-layer feedforward network}

The operation of an ANN consists of the presentation of a set of inputs and subsequent propagation of these inputs through the network. The feedforward three-layer network is selected in our work. The first layer is the input layer which presents data to the network. The nodes in this differ from those in a feedforward network; they are strictly linear in that the output of a node is simply its allowable input. The number of input nodes is calculated from the number of data sources and nodes required to represent each source. Each input node is connected to several nodes in the second layer. The second layer is called the hidden layer, since it is not accessible to the outer environment. The hidden layer acts as a layer of abstraction, pulling features from inputs. Determining the proper number of nodes for the hidden layer is difficult and often determined through experimentation. Generally, the network performance increases with the number of hidden nodes and then reaches a plateau [14]. The addition of more hidden nodes may actually degrade the performance due to increased difficulty of training and the increased potential for overfitting the training data. The implementation of this commonly accepted rule will help train the ANN efficiently and will also help the convergence of the solution. The last layer is referred to as the output layer, since the network's output is the response of nodes in this layer. The number of outputs of an ANN is determined by the neural paradigm used and the type of expected output.

In general, the operation of this feedforward network consists of passing weighted and summed input signals through a chosen nonlinearity. It presumes knowledge of the network's bias functions and weighted links. Once activation and output functions are chosen, an ANN is completely described by its weights and biases. Since a given ANN solves a specific problem, or function, finding weights and biases for the network is equivalent to finding the input/output relationship that describes the function. Thus used to find relationships that are difficult to describe explicitly, ANNs are especially appropriate and powerful because weights and biases can represent a given function.

\subsection{Back-propagation learning rule}

The back-propagation learning rule is an iterative gradient algorithm designed to minimize the mean square error between the actual output of a multilayer feedforward network and the desired output. An essential component of the rule is the iterative method that propagates error terms required to adapt weights back from nodes in the output layer to nodes in the lower layers. 
At the beginning, all weights and node offsets are set to small random values. The input values are presented and the desired outputs are specified. Then the network is used to calculate actual outputs. A recursive algorithm, starting at the output nodes and working back to the hidden layer, adjusts the weights until they converge and the cost function is reduced to an acceptable value. The training process is repeated by presenting different sets of input data to the ANN.

\section{Test studies}

\subsection{Description of the study system}

The system under study is a distribution system in the west of Taipei in 1986. It comprises one substation and ten feeders. As shown in Fig. 1 , the substation is labeled YE and the feeders are labeled YE21, YE22, ..., YE30. The voltage level of each feeder is $11.4 \mathrm{kV}$. The feeder loading data are listed in Table 1.

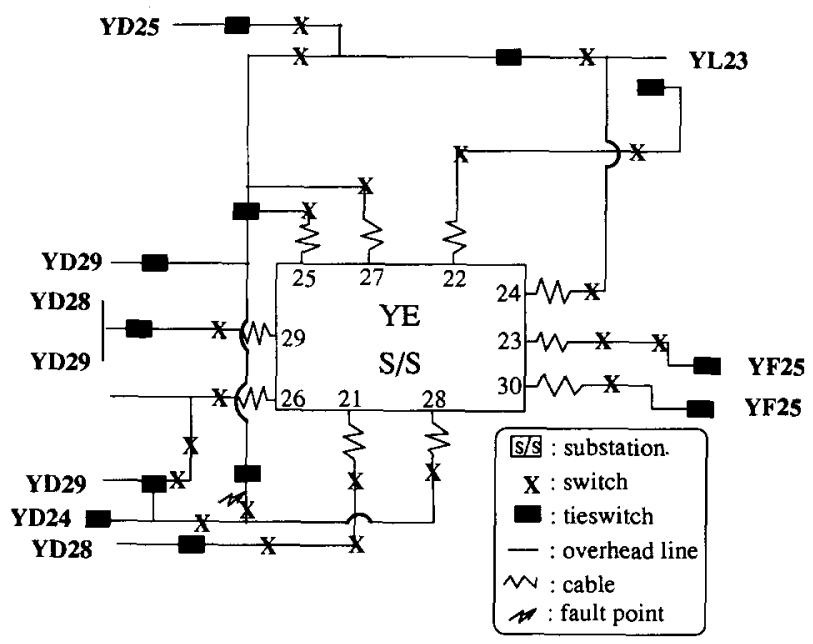

Fig. 1. The study system.

TABLE 1. Load and customer data

\begin{tabular}{lcc}
\hline Feeder & Load (kVA) & $\begin{array}{l}\text { Number of } \\
\text { customers }\end{array}$ \\
\hline 21 & 9729.0 & 432 \\
22 & 10837.4 & 6553 \\
23 & 16940.5 & 6783 \\
24 & 14753.0 & 6688 \\
25 & 2445.0 & 171 \\
26 & 6481.0 & 457 \\
27 & 12561.0 & 989 \\
28 & 5212.0 & 349 \\
29 & 8294.5 & 767 \\
30 & 8215.0 & 1297 \\
\hline
\end{tabular}

\subsection{Reliability indices}

To evaluate the reliability of the test distribution system, the following data are needed:

(1) the failure rates $\lambda$ of all types of components, such as lines, transformers, switches, fuses, and breakers;

(2) the restoration time $r$ of each component;

(3) the switching times $s$ of switches and breakers;

(4) the system configuration;

(5) the load and number of customers at each load point.

With these data at hand, the following two reliability indices are computed [5-7]: the system average interruption frequency index (SAIFI) and the system average interruption duration index (SAIDI).

$$
\begin{aligned}
\text { SAIFI } & =\frac{\text { total no. of customer interruptions }}{\text { total no. of customers served }} \\
& =\frac{\sum \lambda_{i} N_{i}}{\sum N_{i}}
\end{aligned}
$$

where $\lambda_{i}$ is the failure rate and $N_{i}$ is the number of customers at load point $i$.

$$
\begin{aligned}
\text { SAIDI } & =\frac{\text { sum of customer interruption durations }}{\text { total no. of customers served }} \\
& =\frac{\sum U_{i} N_{i}}{\sum N_{i}}
\end{aligned}
$$

where $U_{i}$ is the annual outage time.

\subsection{Experiments and results}

In the experiment, the historical data of the test system from 1982 to 1986 were provided by the Taiwan Power Company. Using these data, the following cases were designed to study the applicability of the ANN approach.

Case 1. Prediction of the reliability indices of 1986 by feeding the ANN with the sytem data from 1982 to 1985 .

Case 2. Evaluation of the reliability indices when the feeder YE28 is overloaded and part of its load is switched to YE25.

Case 3. Evaluation of the reliability indices when a fault occurs in feeder YE28. The fault location is indicated by the arrow in Fig. 1.

The developed ANN is shown in Fig. 2. The data of feeders YE21, YE22, ..., YE30 enter the network from the input layer. The output layer gives the values of the SAIFI and SAIDI of the whole system and each feeder. Corresponding to 


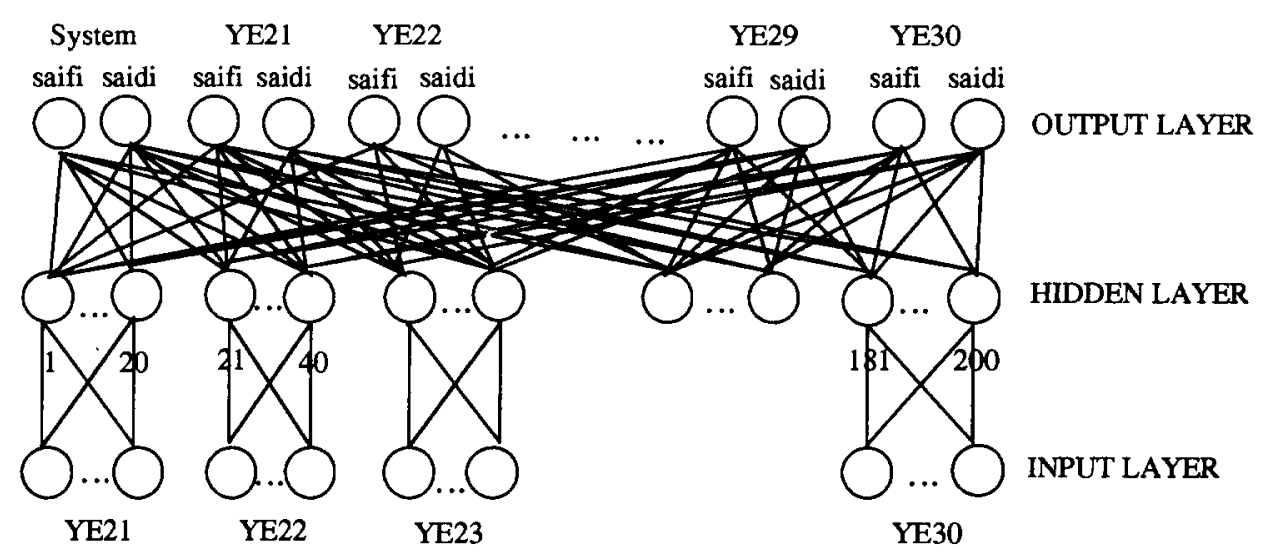

Fig. 2. The constructed neural network.

the number of lines, buses, circuit breakers, and transformers of each feeder, this network contains 3617 nodes in the input layer, 22 nodes in the output layer, and 200 nodes in the hidden layer.

The SAIFI and SAIDI values of YE28, YE25, and the whole system are given in Tables 2 and 3 , respectively. The values computed by the ANN are compared with results from the network method. The deviations are also listed in the Tables. It was found that the deviations of the values computed by the proposed approach from those by the network method are below $1 \%$. The required running time on a SUN network environment is less than $2 \mathrm{~s}$, and the time required for the network method is 3.5 times greater than that of the ANN approach.

\section{Conclusions}

An artificial neural network approach is presented in this paper for evaluating the reliability

TABLE 2. SAIDI values

\begin{tabular}{|c|c|c|c|c|c|c|c|c|c|}
\hline & \multicolumn{3}{|l|}{ YE28 } & \multicolumn{3}{|l|}{ YE25 } & \multicolumn{3}{|c|}{ System } \\
\hline & $\mathrm{A}$ & B & Deviation (\%) & A & $\mathrm{B}$ & Deviation (\%) & A & $\mathrm{B}$ & Deviation $(\%)$ \\
\hline 1982 & 0.2584 & 0.2586 & 0.08 & 0.2845 & 0.2859 & 0.50 & 0.7868 & 0.7869 & 0.01 \\
\hline 1983 & 0.2987 & 0.2986 & 0.03 & 0.3641 & 0.3635 & 0.20 & 0.9051 & 0.9051 & 0.00 \\
\hline 1984 & 0.2383 & 0.2382 & 0.04 & 0.2740 & 0.2726 & 0.50 & 0.7417 & 0.7417 & 0.00 \\
\hline 1985 & 0.3513 & 0.3515 & 0.06 & 0.3956 & 0.3954 & 0.05 & 1.0415 & 1.0415 & 0.00 \\
\hline Case 1 & 0.1654 & 0.1647 & 0.40 & 0.1466 & 0.1478 & 0.80 & 0.5084 & 0.5084 & 0.00 \\
\hline Case 2 & 0.1516 & 0.1509 & 0.50 & 0.1648 & 0.1658 & 0.60 & 0.5084 & 0.5082 & 0.04 \\
\hline Case 3 & 0.1526 & 0.1521 & 0.30 & 0.1466 & 0.1478 & 0.80 & 0.5081 & 0.5084 & 1.06 \\
\hline
\end{tabular}

$\mathrm{A}=$ network method; $\mathrm{B}=$ neural network approach.

TABLE 3. SAIFI values

\begin{tabular}{|c|c|c|c|c|c|c|c|c|c|}
\hline & \multicolumn{3}{|l|}{ YE28 } & \multicolumn{3}{|l|}{ YE25 } & \multicolumn{3}{|c|}{ System } \\
\hline & $\mathrm{A}$ & $\mathrm{B}$ & Deviation $(\%)$ & $\mathrm{A}$ & B & Deviation $(\%)$ & $\mathrm{A}$ & $\mathbf{B}$ & Deviation $(\%)$ \\
\hline 1982 & 0.3907 & 0.3897 & 0.30 & 0.2991 & 0.2989 & 0.07 & 1.2580 & 1.2580 & 0.00 \\
\hline 1983 & 0.4210 & 0.4209 & 0.02 & 0.3356 & 0.3341 & 0.50 & 1.3672 & 1.3672 & 0.00 \\
\hline 1984 & 0.3696 & 0.3713 & 0.50 & 0.3040 & 0.3056 & 0.50 & 1.2146 & 1.2148 & 0.02 \\
\hline 1985 & 0.4816 & 0.4840 & 0.50 & 0.3455 & 0.3453 & 0.06 & 1.4006 & 1.4002 & 0.03 \\
\hline Case 1 & 0.2906 & 0.2922 & 0.60 & 0.2112 & 0.2108 & 0.20 & 0.7521 & 0.7526 & 0.07 \\
\hline Case 2 & 0.2643 & 0.2649 & 0.20 & 0.2450 & 0.2441 & 0.40 & 0.7524 & 0.7518 & 0.08 \\
\hline Case 3 & 0.2954 & 0.2942 & 0.40 & 0.2112 & 0.2108 & 0.20 & 0.7523 & 0.7520 & 0.04 \\
\hline
\end{tabular}

$\mathrm{A}=$ network method; $\mathrm{B}=$ neural network approach. 
of distribution systems and a three-layer feedforward network with the back-propagation learning rule is constructed. The network contains 3617 nodes in the input layer, 200 nodes in the hidden layer, and 22 nodes in the output layer. The proposed neural network approach was tested on a real distribution system in the west of Taipei. Three cases were designed to demonstrate the capability of the developed neural network. With sufficient learning from historical data, the reliability indices of the normal, overloaded, and faulted conditions are computed with tiny deviations, below $1 \%$, from the results by the network method. The required computing time is less than $2 \mathrm{~s}$. In view of the effectiveness and efficiency of the proposed approach, the developed artificial neural network may become a valuable tool to assist distribution system operators in evaluating system reliability.

\section{References}

1 Y. Y. Hsu, J. L. Chen and L. M. Chen, Development of a software program for the evaluation of distribution system reliability, Res. Rep., Taiwan Power Company, June 1988.

2 P. A. Jensen and M. Bellmore, An algorithm to determine the reliability of a complex system, IEEE Trans. Reliability, 18 (1969) 169-174.
3 J. Endrenyi, P. C. Maenhaut and L. E. Payne, Reliability evaluation of transmission systems with switching after fault--approximation and computer program, IEEE Trans., PAS-92 (1973) 1863-1875.

4 R. N. Allan, R. Billinton and M. F. De Oliveira, An efficient algorithm for deducing the minimal cuts and reliability indices of a general network configuration, IEEE Trans. Reliability, 25 (1976) 226-233.

5 R. Billinton and R. N. Allan, Reliability Evaluation of Power Systems, Plenum Press, New York, 1984.

6 J. Endrenyi, Reliability Modeling in Electric Power Systems, Wiley, New York, 1978.

7 Development of distribution system reliability and risk analysis models, EPRI Rep. No. EL-2018, Electr. Power Res. Inst., Palo Alto, CA, 1981.

8 R. P. Lippman, An introduction to computing with neural nets, IEEE ASSP Mag., 4 (1987) 4-22.

9 Y. H. Pao, Adaptive Pattern Recognition and Neural Networks, Adison-Wesley, Reading, MA, 1989.

$10 \mathrm{~T}$. Kohonen, Self-Organization and Associative Memory, Springer, Berlin, 1984.

11 D. E. Rumelhart, G. E. Hinton and R. J. Williams, Learning internal representations by error propagation, parallel distributed processing, Explorations in the Microstructures of Cognition, Vol. 1: Foundations, MIT Press, Cambridge, MA, 1986, pp. $318-362$.

12 D. E. Rumelhart, G. E. Hinton and R. J. Williams, Learning representations by back-propagating errors, Nature (London), 323 (1986) 696-699.

13 D. Bailey and D. Thompson, How to develop neural-network applications, AI Expert, 5 (June) (1990) 39-47.

14 W. M. Huang and R. P. Lippman, Comparisons between neural networks and conventional classifiers, Proc. IEEE Int. Conf. Neural Networks, San Diego, CA, USA, 1987, pp. $485-493$. 